\title{
A COLLECTION OF CASIM CALCULATIONS
}

J.D. Cossairt

October 22, 1982

Monte Carlo calculations of hadronic cascades at Fermilab have usually been done using the code CASIM written by A.VanGinneken. ${ }^{1,2}$ These calculations are often performed to determine the quantity of shielding required for radiation protection purposes. A number of examples of such calculations are presented in Ref.1. Several years of practical experience have led the author to develop the collection of additional cases included in the present report. It is hoped that these results along with those given in Ref. 1 will serve as a useful reference. No attempt was made here to consider "a11" possibilities; rather, the purpose was to develop a useful set of examples. Exceptionally intricate cases should, of course, receive individualized attention as appropriate.

For these examples, the FORTRAN-V version of CASIM as modified for the Fermilab-CYBER system was used in all cases. As is customary with this version, elastic scattering(Coulomb and nuclear diffractive) were included in the calculations. The usual lower momentum cutoff of $0.3 \mathrm{GeV} / \mathrm{c}$ was employed so that all conversion factors used to convert stars $/ \mathrm{cm}^{3}$ to $\mathrm{mrad}$ or mrem described in Ref. 1 and also by Peter Gollon ${ }^{3}$ would be valid. An additional feature of this version of the program used here is that the incident beam profile is a Gaussian. A11 calculations were made using protons as the incident beam. Experience indicates the choice of incident hadron makes little difference. Several different materials were used in the calculations presented here. As is described elsewhere, ${ }^{4}$ it appears that a density of 2.24 grams $/ \mathrm{cm}^{2}$ is appropriate for soil compacted into a berm. Concrete was chosen to have a density of $2.4 \mathrm{grams} / \mathrm{cm}^{2}$ and the same average atomic number(11) and atomic weight(2.3) as the soil. Where no material is indicated, vacuum is assumed. These calculations were al1 performed using cylindrical symmetry in order to keep the geometry simple. The 
results are shown as contour plots of equal star density per incident proton as a function of $\operatorname{depth}(Z)$ and radius(R) superimposed upon a scale drawing of the situation under consideration. It should be noted that both feet and $\mathrm{cm}$ scales are given and that the abscessia and ordinate scales are not the same. The figures hopefully have enough information on them to be largely self-explanatory. Descriptions of groups of figures follow below giving additional commentary where necessary. The author will maintain the original printouts on file if more detailed reference to them is desired. 
Group I - Figures 1-8

Case of a Magnet in an Enclosure

Here a magnet very similar in cross section to a $10 \mathrm{ft}$ long EPB dipole or Energy Saver dipole, was modeled having rectangular dimensions ( $3.3 \times 12.7 \mathrm{~cm}$ aperture and $31.8 \times 40.6 \mathrm{~cm}$ outside). No allowance was made for the vacuum can. The beam was brought in "head on" to the magnet one value of $\sigma$ deep into one of the pole pieces. The beam was centered on the magnet and no magnetic field was used. These cases are useful to get worst case estimates since all situations involving "scraping" losses on the magnet would be somewhat lower. Scraping loss cases were not attempted here since they are so dependent upon individual beam profiles. Figures 5-8 show the effects of a $2 \mathrm{ft}$ thick steel shield installed both downstream of the magnet and directly over it in order to show the effect of the placement of steel as it is used to gain economy of space in typical situations. The figures with pure soil shields show two different enclosure radii; $3 \mathrm{ft}$ and $6 \mathrm{ft}$. If one does the calculation for a $20 \mathrm{ft}$ long magnet, the contours do not move outward; rather the region of peak dose is merely extended.

Group II - Figures 9-12 -

Case of a Thin Beam Pipe in an Enclosure

In these four figures are shown contour plots for a 4-inch(outside) diameter aluminum beam pipe having $1 / 8$ inch thick walls. The beam was modeled to be centered on the pipe one $\mathrm{nm}$ from the inner wall of the pipe. Again, scraping loss cases would be less severe so that this situation is again conservative. A beam as small as $\sigma=2 \mathrm{~cm}$ is also a rather severe assumption for normal beam transport lines. Again two different enclosure radii are used.

Group III - Figures 13-16

Thick Steel Pipes in an Enclosure

These are similar to the situation of Group II except that a one $\mathrm{ft}$ diameter steel pipe with $\frac{1}{2}$ inch thick walls is the target. The beam hits the pipe head-on one value of sigma from the inner wall. 
Group IV - Figures 17-20

Steel Pipe Buried in Soil

The same pipe considered as the target in Group III is now buried in soil. The targeting conditions are the same as before. Figures 19 and 20 show the effect of $3 \mathrm{ft}$ of steel shielding over the pipe. If one considers scraping losses, the same peak star density is obtained at a given value of $R$ but the value of $Z$ for the shower maximum is a function of the details of the targeting conditions as long as most of the beam eventually interacts in the soil. of course, for short runs of beam pipe, very small scraping angle, and large beam sizes, this may be important. Such cases should be individually treated. 
A.Van Ginneken and M.Awschalom

"High Energy Particle Interactions in Large Targets", Fermilab, 1975.

2 A.Van Ginneken

"CASIM - Program to Simulate Transport of Hadronic Cascades in Bulk Matter", FN-272, January 1975.

3

Peter J. Gollon

"Dosimetry and Shielding Factors Relevant to the Design of Iron Beam Dumps", TM-664, March 1976.

4

J.D.Cossairt, N.V.Mokho, and C.T.Murphy,

Nuclear Instruments and Methods, 197(1982) 465 


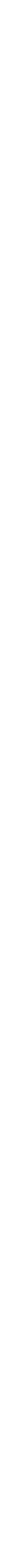




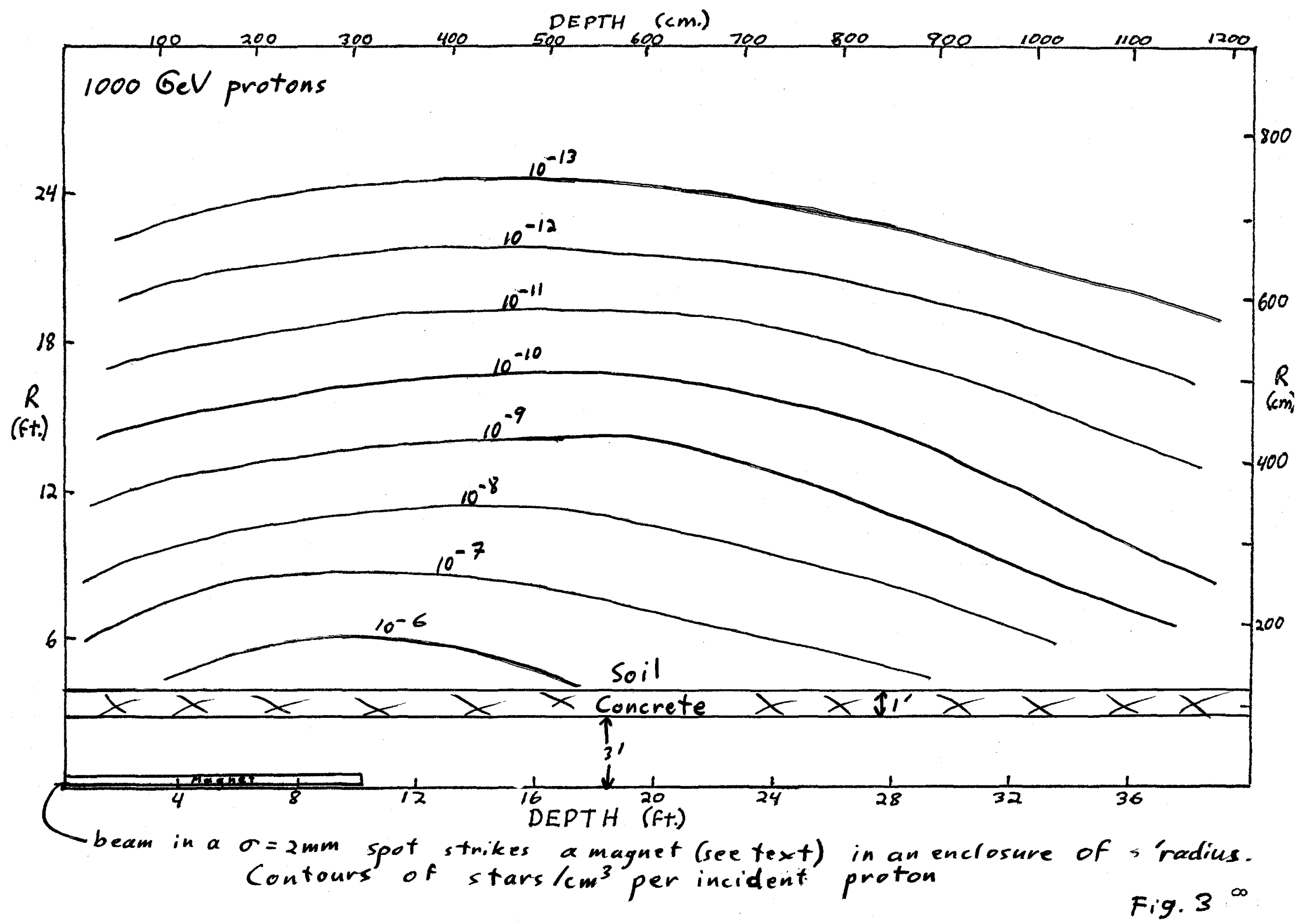




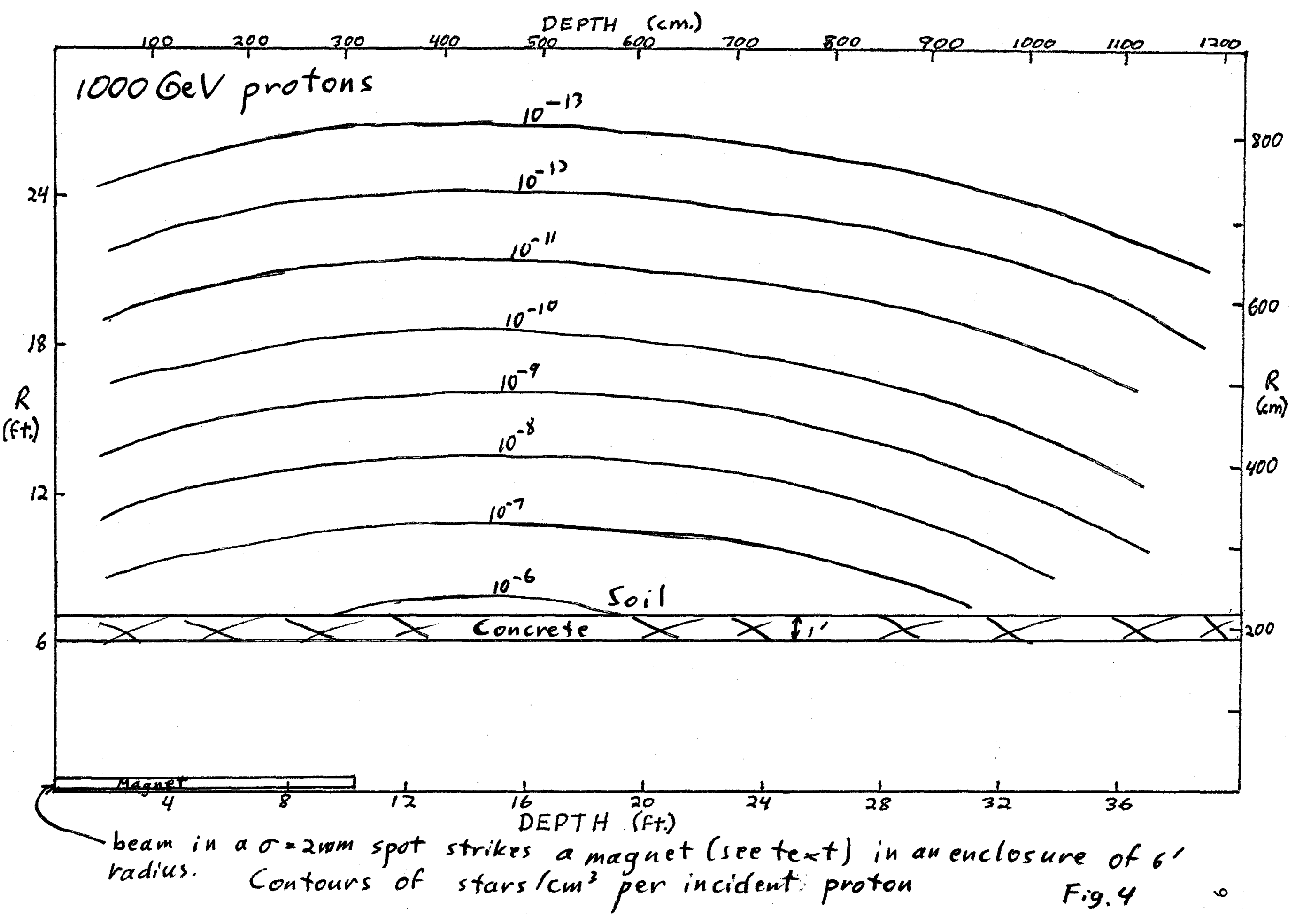




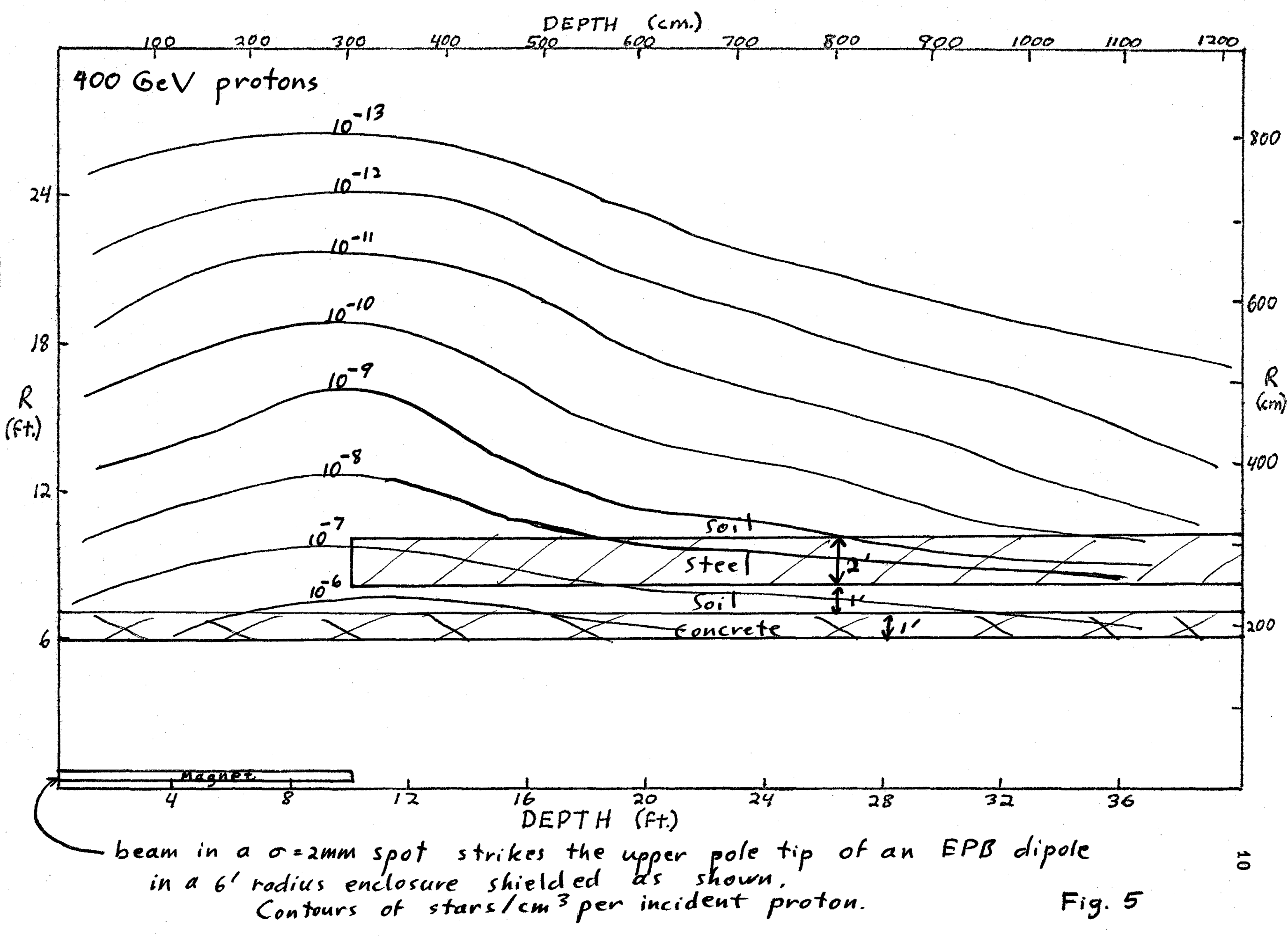




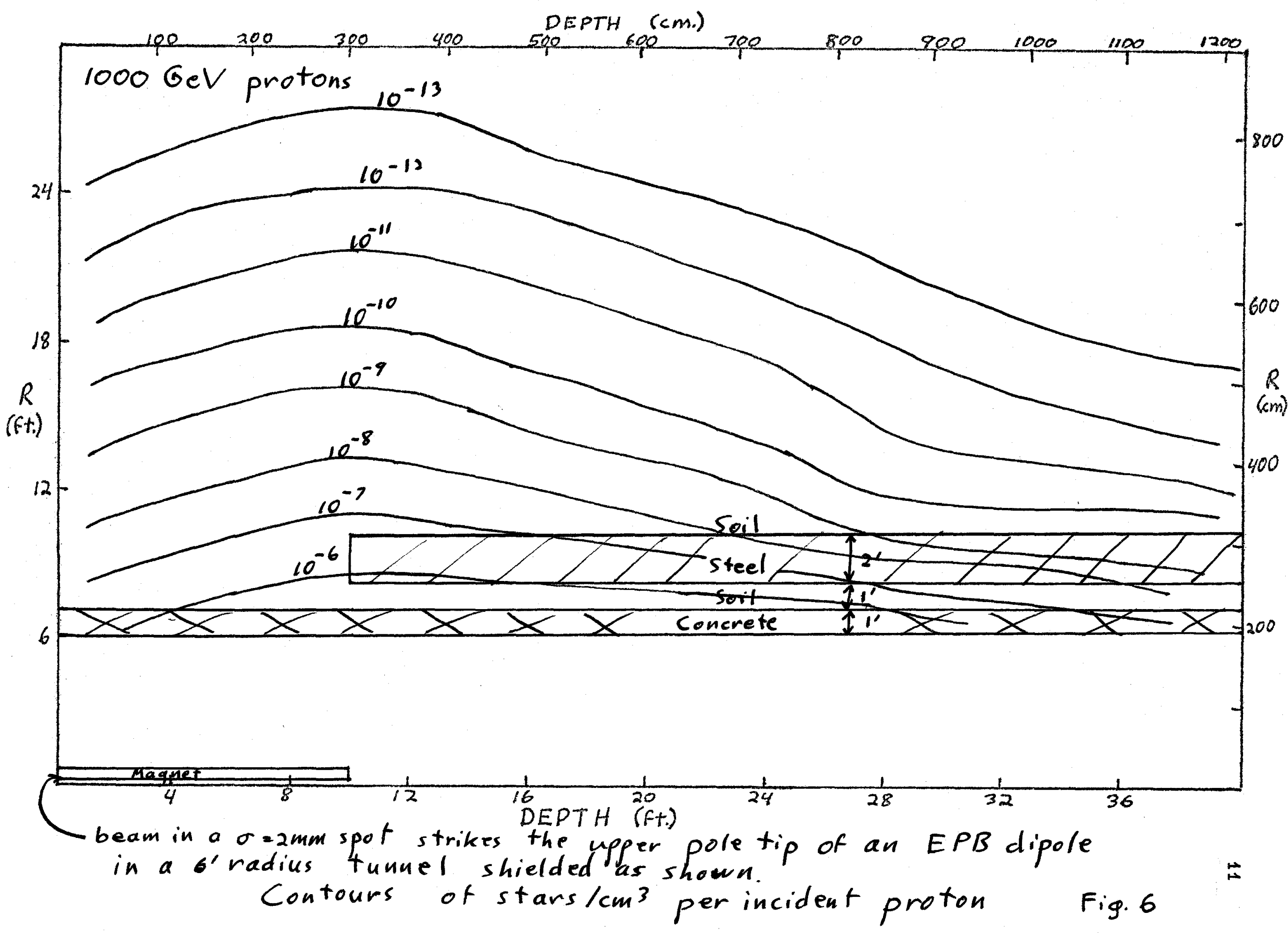




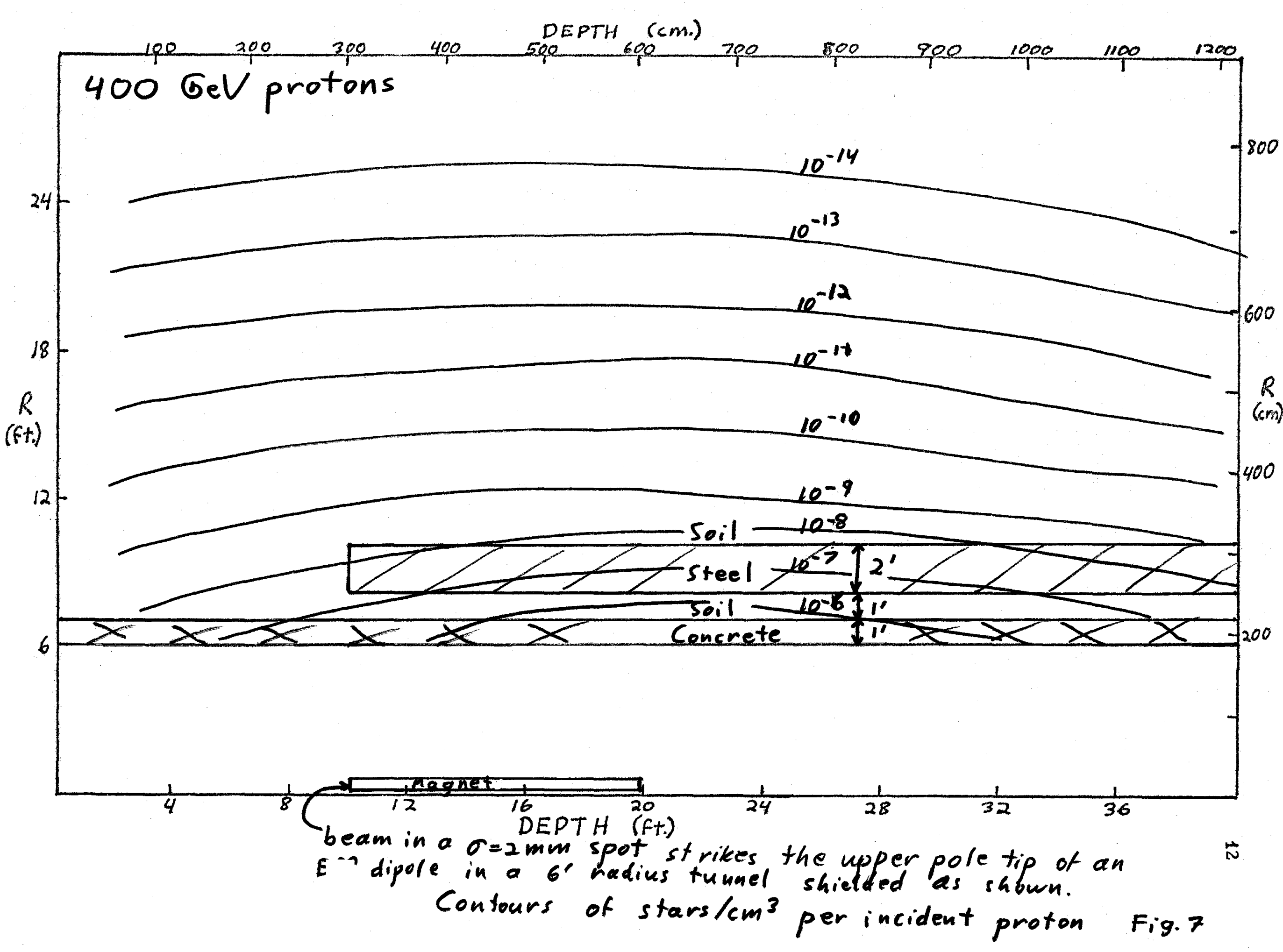




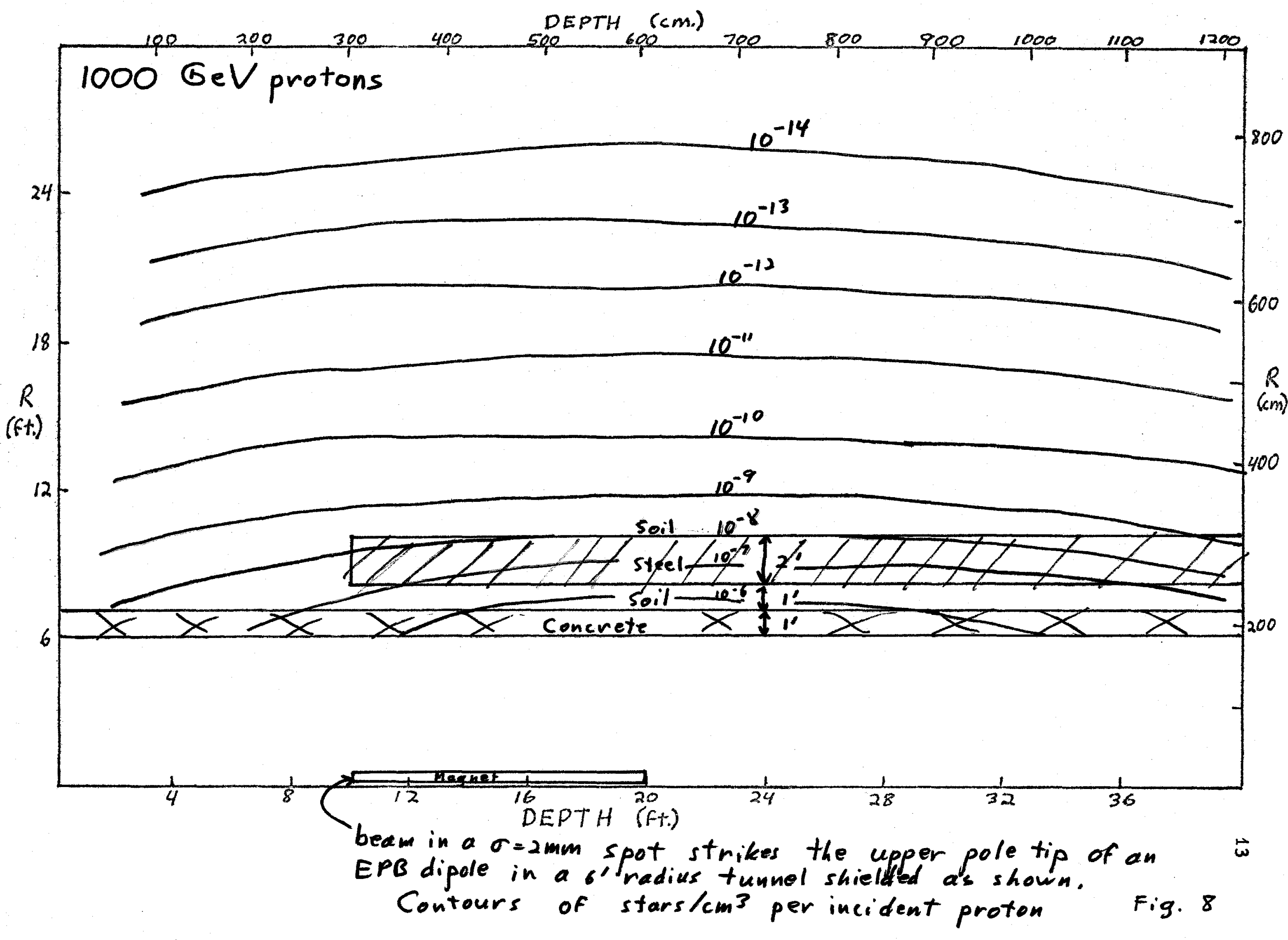




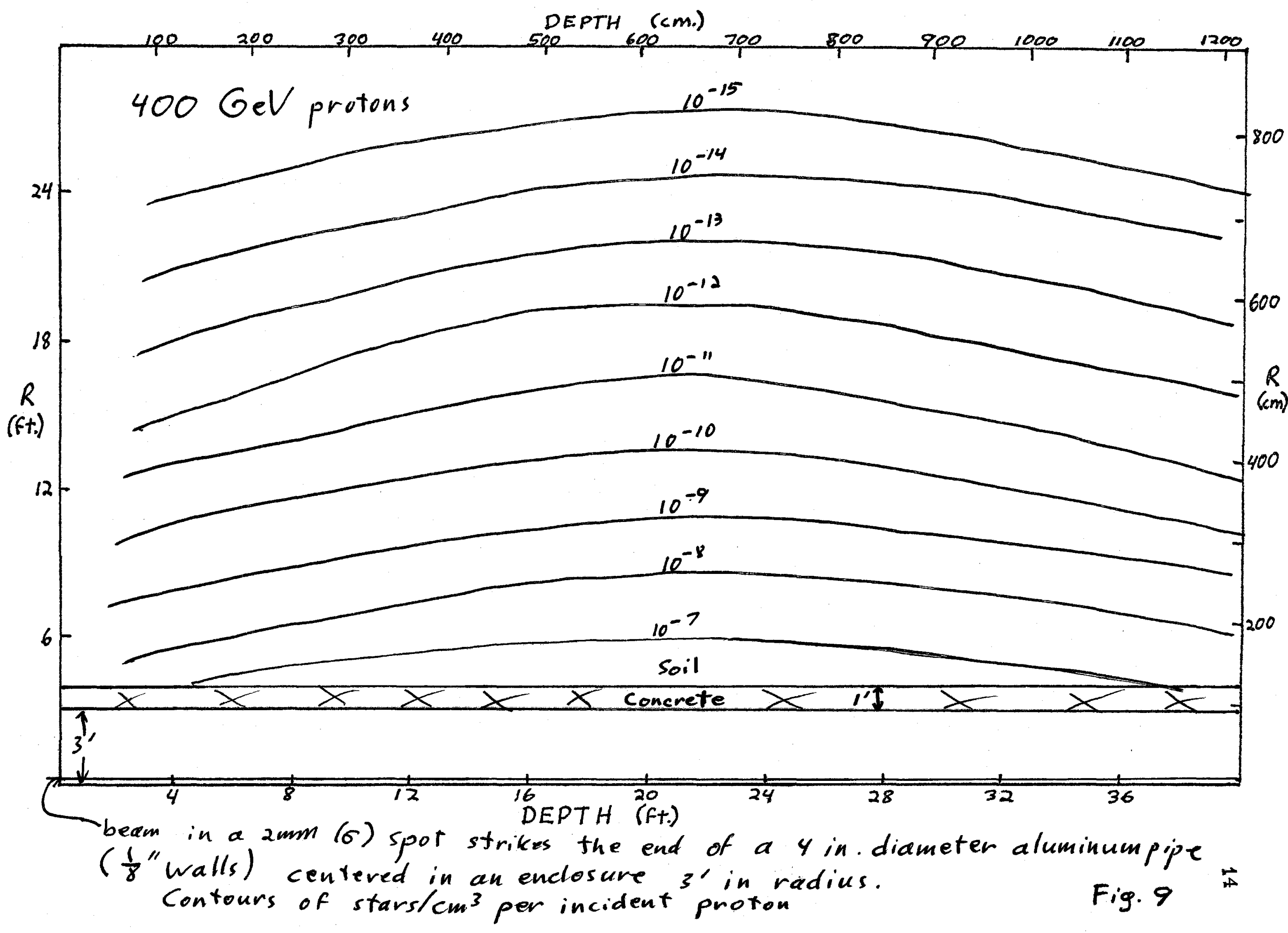




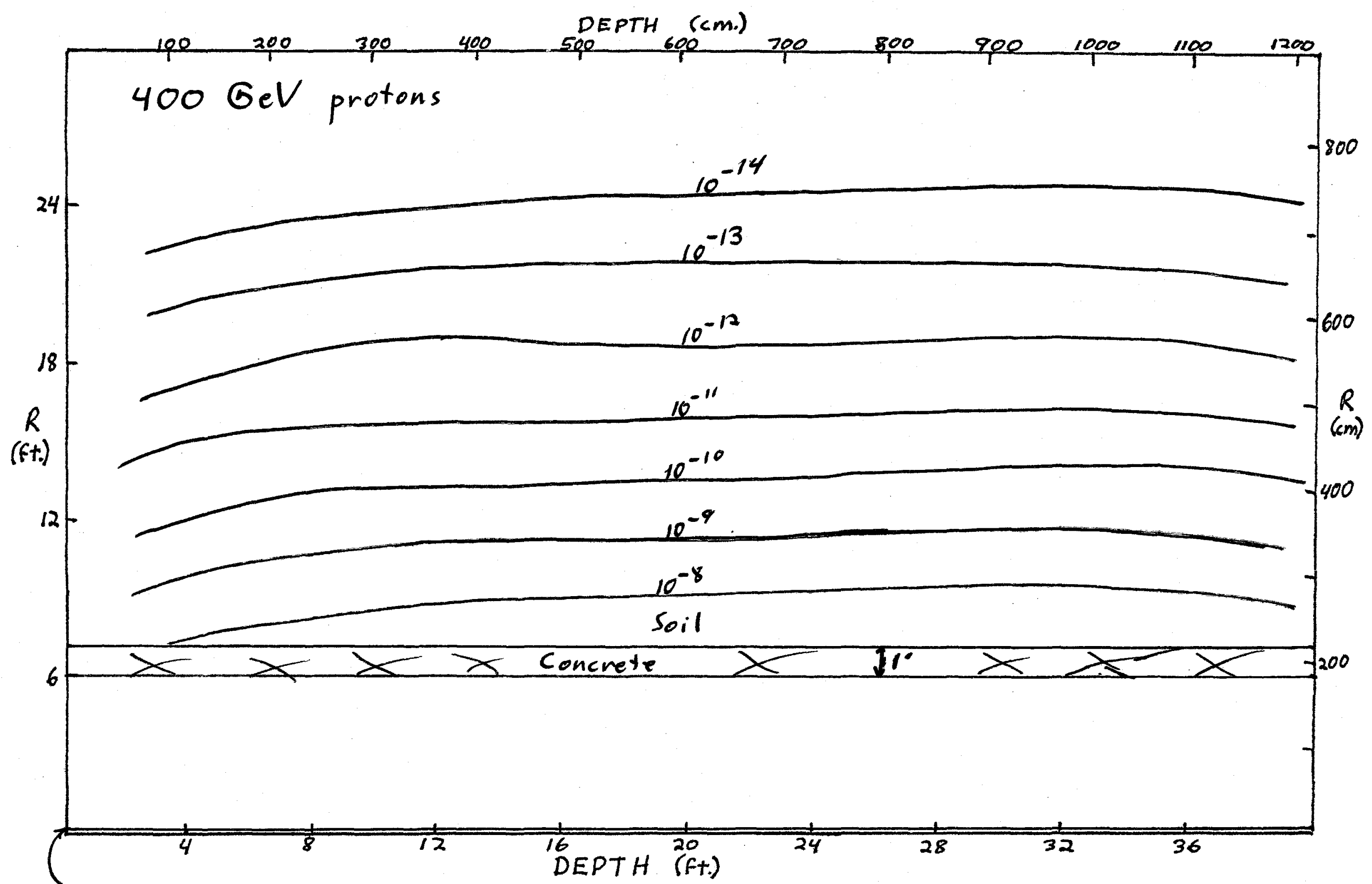

beam in a $q=2 \mathrm{~mm}$ spot strikes the end of a 4 in. diameter aluminum pipe ( $\frac{1}{8}$ "walls) centered in an enclosure $6^{\prime}$ in radius', Contours of stars/ $\mathrm{cm}^{3}$ per incident proton 


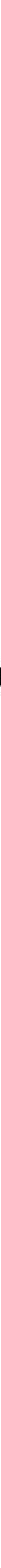




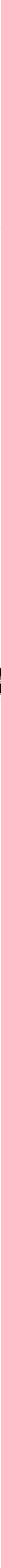




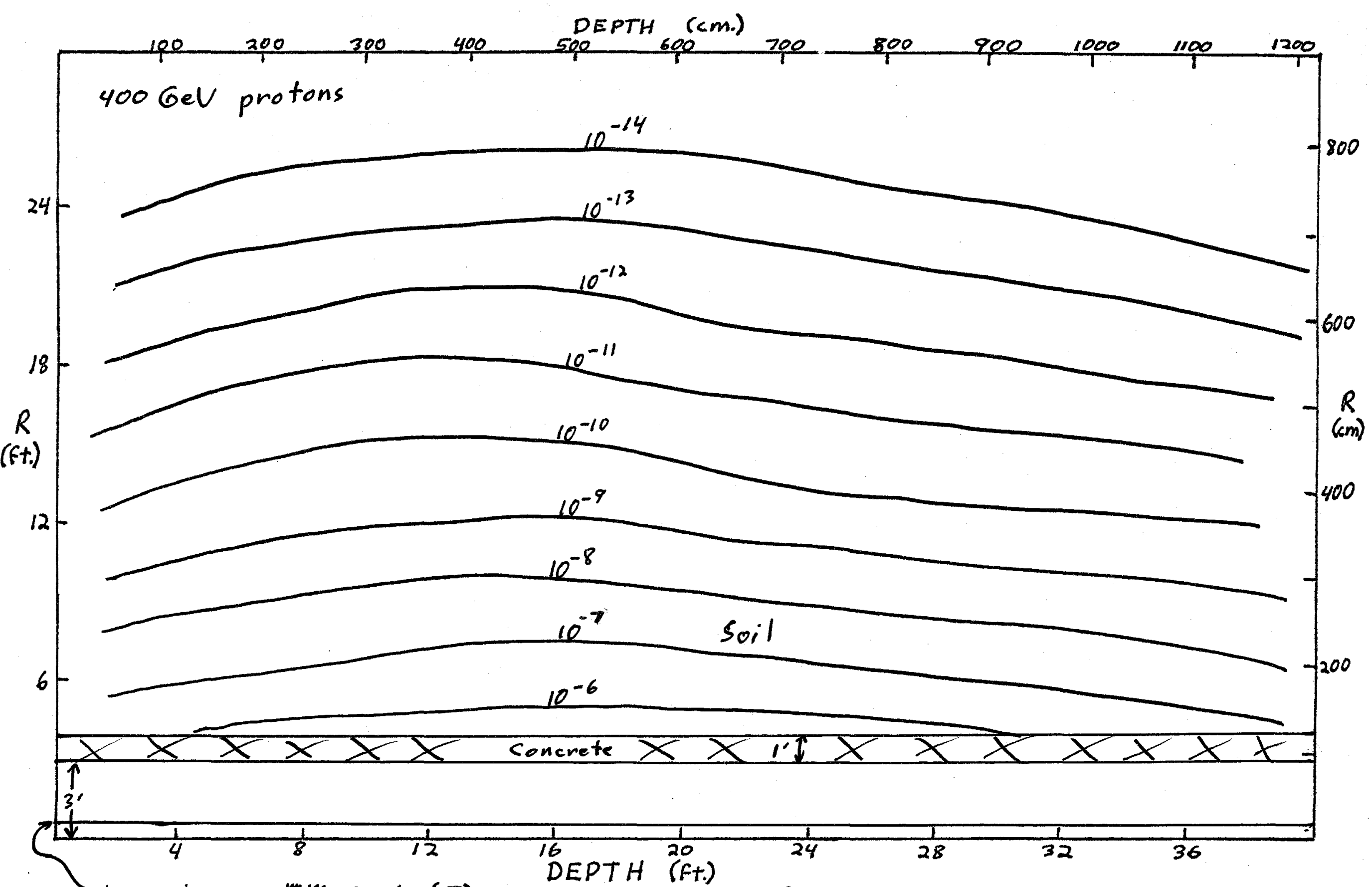
beam in a $2 \mathrm{~mm}$ spot $(\sigma)$ strikes the end of a 1 ft diameter pipe (strell
$\left(\frac{1}{2}\right.$ "walls) centered in an enclosure $3^{\prime}$ in radius. Contours of stars $/ \mathrm{cm}^{3}$ per incident proton

Fig. 13 


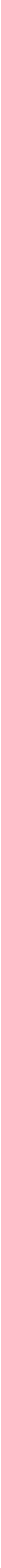




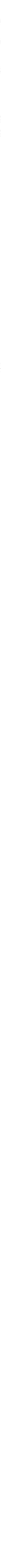

beam in a $\sigma=2 \mathrm{~mm}$ spot strikes the end of a Ift. diameter pipe (steel) ( $\frac{1}{2}$ "walls) cintered in an enclosume $3^{\prime}$ in radius. Contours of stars $1 \mathrm{~cm}^{3}$ per incident proton 


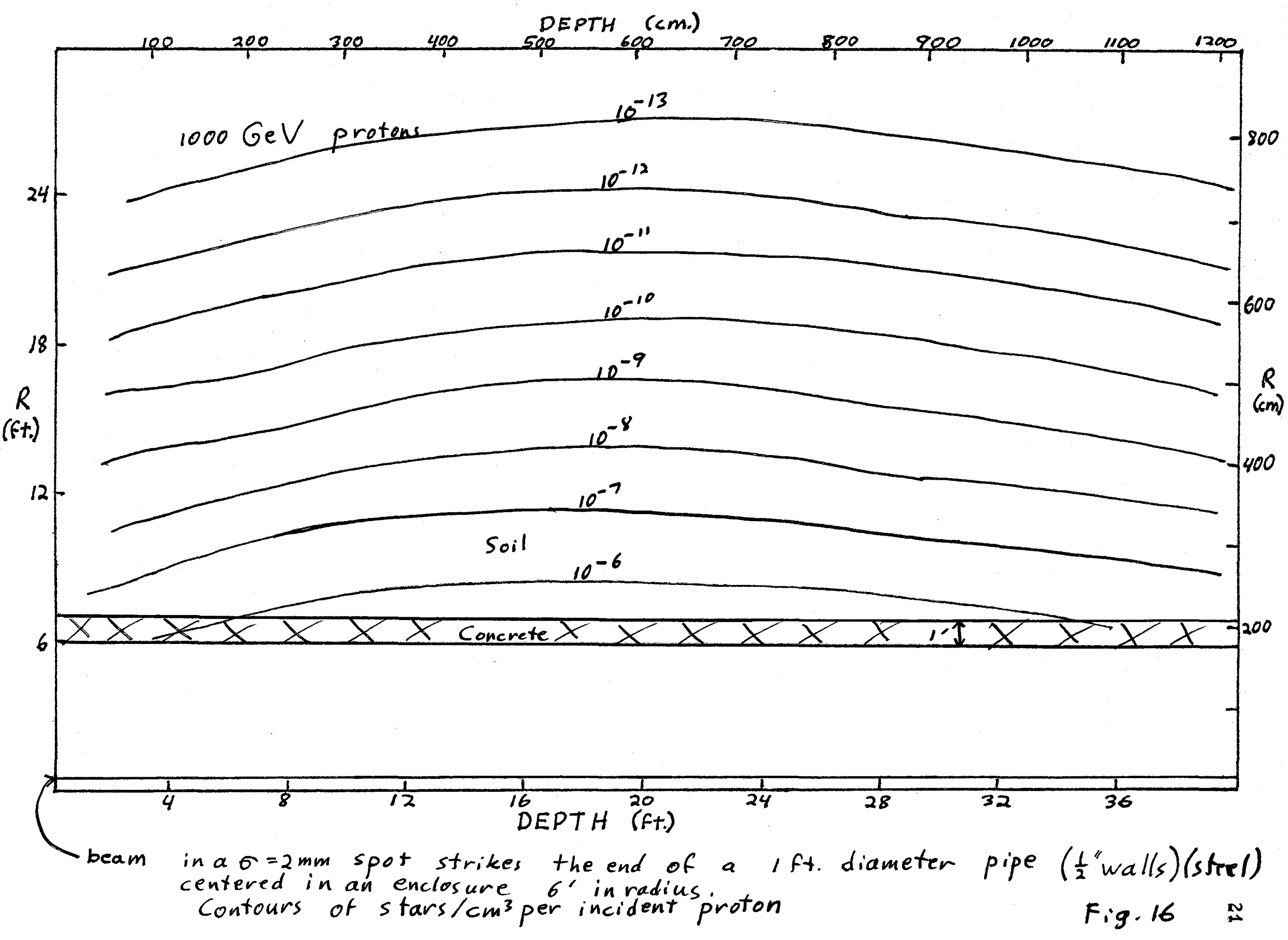




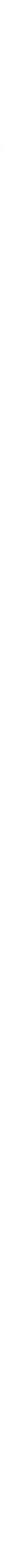




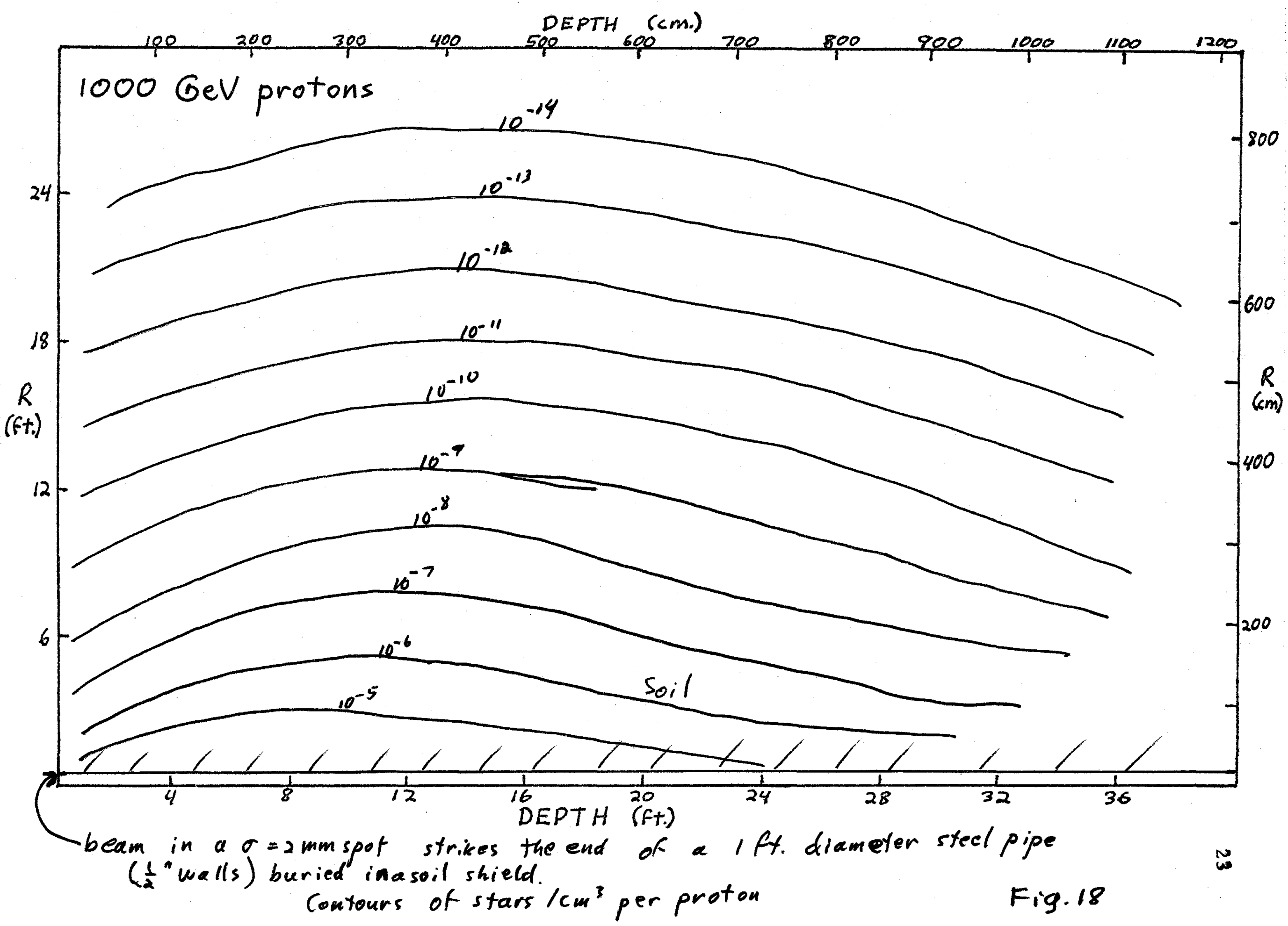




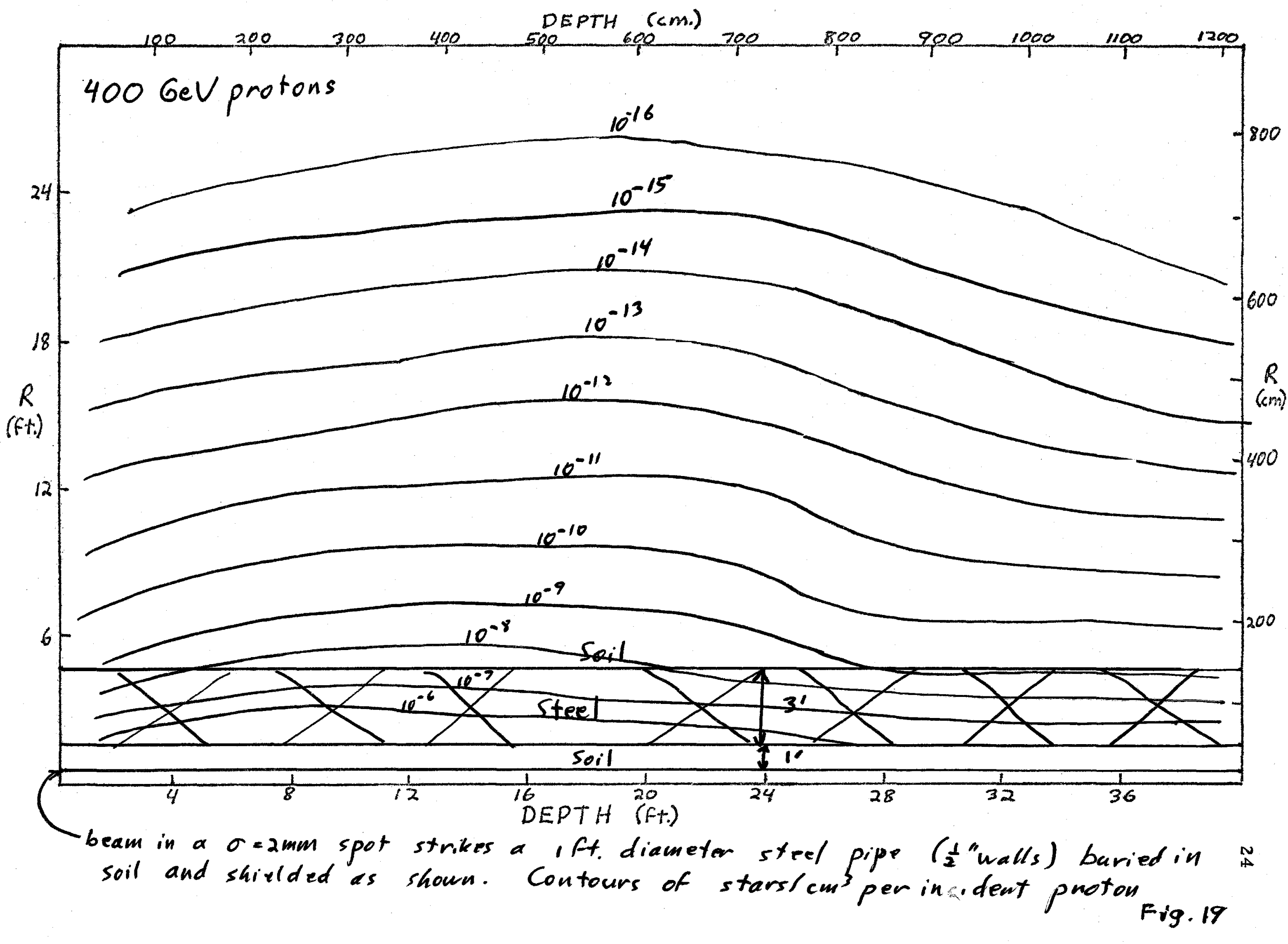


